\title{
Clinical Study \\ Effects of Water Extracts of Graptopetalum paraguayense on Blood Pressure, Fasting Glucose, and Lipid Profiles of Subjects with Metabolic Syndrome
}

\author{
Chi-Hua Yen, ${ }^{1,2,3}$ Shu-Ju Chen, ${ }^{4}$ Jen-Tzu Liu, ${ }^{5}$ Yu-Fen Tseng, ${ }^{5}$ and Ping-Ting Lin ${ }^{5,6}$ \\ ${ }^{1}$ Department of Family and Community Medicine, Chung Shan Medical University Hospital, Taichung 40201, Taiwan \\ ${ }^{2}$ School of Medicine, Chung Shan Medical University, Taichung 40201, Taiwan \\ ${ }^{3}$ Center for Education and Research on Geriatrics and Gerontology, Chung Shan Medical University, Taichung 40201, Taiwan \\ ${ }^{4}$ Department of Health Food, Chung Chou University of Science and Technology, Changhua 51003, Taiwan \\ ${ }^{5}$ School of Nutrition, Chung Shan Medical University, Taichung 40201, Taiwan \\ ${ }^{6}$ Department of Nutrition, Chung Shan Medical University Hospital, Taichung 40201, Taiwan
}

Correspondence should be addressed to Ping-Ting Lin; apt810@csmu.edu.tw

Received 16 September 2013; Accepted 29 October 2013

Academic Editor: Esteban Martinez

Copyright (C) 2013 Chi-Hua Yen et al. This is an open access article distributed under the Creative Commons Attribution License, which permits unrestricted use, distribution, and reproduction in any medium, provided the original work is properly cited.

This study was aimed to investigate the effects of water extracts of Graptopetalum paraguayense (WGP, $4 \mathrm{~g} / \mathrm{d}$ ) on blood pressure, blood glucose level, and lipid profiles in subjects with metabolic syndrome (MS). Participants with MS $(n=54)$ were randomly assigned to the placebo $(n=28)$ and WGP groups $(n=26)$, and the intervention was administered for 12 weeks. Systolic blood pressure (SBP), diastolic blood pressure (DBP), fasting glucose (FG), lipid profiles (total cholesterol (TC), triglyceride (TG), low density lipoprotein cholesterol (LDL-C), and high density lipoprotein (HDL-C)), and antioxidant enzymes activities (catalase (CAT), superoxide dismutase (SOD), and glutathione peroxidase (GPx)) were measured. Forty-two subjects completed the study (placebo, $n=19$; WGP, $n=23$ ). FG, SBP, and LDL-C levels were significantly lower and HDL-C level and antioxidant enzymes activities (CAT and SOD) were significantly higher after WGP supplementation. Blood pressure, FG, and lipid profiles were significantly correlated with antioxidant enzymes activities after supplementation $(P<0.05)$. The present study demonstrated a significant reduction in blood pressure, blood glucose, and lipid profiles and an increase in antioxidant enzymes activities in subjects with MS after WGP supplementation. Taken together, the antioxidative capacity of WGP might exert a beneficial effect on MS. This trial is registered with ClinicalTrials.gov NCT01463748.

\section{Introduction}

Metabolic syndrome (MS) represents a collection of physiological and anthropometric abnormalities [1] and is recognized as a significant risk factor for cardiovascular disease and type II diabetes [2]. The National Health and Nutrition Examination Surveys (NHANES) indicated that more than $30 \%$ of the adult population in the USA suffered from MS [3-5]. In Taiwan, reports from the Nutrition and Health Survey in Taiwan (NAHSIT) indicated that the prevalence of MS in adults ( $\geq 18 \mathrm{y}$ ) was $20 \%$ during $1993-$ 1996 and increased to $28.5 \%$ during 2005-2008 [6]. The characteristics of MS, including insulin resistance, type II diabetes, hypertension, dyslipidemia, and visceral obesity, may increase oxidative stress [7-9] and reduce antioxidant defenses [10-12]. Increases in oxidative stress contribute to impaired vascular function, thrombosis, and atherosclerosis that ultimately result in vascular disease [13].

Graptopetalum paraguayense E. Walther is a popular traditional Chinese herbal medicine that belongs to the Crassulaceae family. Several biological activities of Graptopetalum paraguayense have been reported, including antityrosine [14], radical-scavenging [15], antihypertensive [16, 17], and hepatoprotective effects [18-20], in addition to protection against brain injury in ischemic rats [21]. Most studies have investigated the functions of Graptopetalum paraguayense in cell 
or animals models [14-21]. To our knowledge, Lin et al. [22] conducted the only clinical study where subjects with hypercholesterolemia consumed Graptopetalum paraguayense as a serving of vegetable $(100 \mathrm{~g} / \mathrm{d})$ for 8 weeks and found a significant decrease in the level of oxidative stress but no change in lipid profiles. These results may be because of the suboptimal Graptopetalum paraguayense dosage and short intervention duration. Therefore, this study was aimed to examine the effects of a 12-week supplementation with water extracts of Graptopetalum paraguayense (WGP) on blood pressure, fasting glucose (FG), and lipid profiles of subjects with MS.

\section{Materials and Methods}

2.1. Participants. Individuals with MS were recruited from the Department of Family and Community Medicine at the Chung Shan Medical University Hospital in Taiwan. The inclusion criteria for MS in adults were based on the guidelines of the Administration of Health Promotion, Ministry of Health and Welfare, Taiwan (2007). Subjects were considered to have MS if they had three of the following five characteristics: (1) abdominal obesity (waist circumference $\geq 90 \mathrm{~cm}$ in men and $\geq 80 \mathrm{~cm}$ in women), (2) impaired FG ( $\geq 5.6 \mathrm{mmol} / \mathrm{L}$ ), (3) hypertriglyceridemia (triglyceride (TG) $\geq 1.7 \mathrm{mmol} / \mathrm{L}$ ), (4) low high density lipoprotein cholesterol (HDL-C) $<1.0 \mathrm{mmol} / \mathrm{L}$ in men and $<1.3 \mathrm{mmol} / \mathrm{L}$ in women, and (5) increased blood pressure (systolic blood pressure (SBP) $\geq 130 \mathrm{mmHg}$ and diastolic blood pressure (DBP) $\geq 85 \mathrm{mmHg}$ ). Subjects using antidiabetic, antihypertensive, and lipid-lowering medications were considered to have elevated FG, elevated blood pressure, and dyslipidemia, respectively. The diagnostic criteria for MS in Taiwan are in accordance with the National Cholesterol Education Program Adult Treatment Panel III (ATP III, 2001), International Diabetes Federation (IDF, 2005), and the American Heart Association/National Heart, Lung, and Blood Institute (AHA/NHLBI, 2005) criteria $[1,23,24]$. Therefore, the diagnostic criteria for MS in Taiwan are similar with these international criteria. This study was approved by the Institutional Review Board of the Chung Shan Medical Hospital in Taiwan, and the written informed consent was obtained from each subject. The trial was registered at ClinicalTrials.gov (no. NCT01463748).

\subsection{Administration of Water Extracts of Graptopetalum para-} guayense (WGP). Fresh Graptopetalum paraguayense E. Walther was purchased from a local organic farm (TOPF0024, Yunlin County, Taiwan). The dose $(4 \mathrm{~g} / \mathrm{d})$ of the Graptopetalum paraguayense administered was chosen according to the report by Lin et al. [22]. The water extraction rate of Graptopetalum paraguayense was 4\% [14], and as a result, $100 \mathrm{~g}$ of Graptopetalum paraguayense provides $4 \mathrm{~g}$ WGP. The safety of the WGP dose was assessed by Chung et al. [25], and WGP is considered safe with a no observed adverse effect level (NOAEL) of $1.0 \mathrm{~g} / \mathrm{kg}$ body weight in rats, which is equivalent to a daily dose of $0.16 \mathrm{~g} / \mathrm{kg}$ of body weight in humans. The WGP and placebo (starch) capsules were prepared by the Gold Chia Fong Biotech Co., Ltd. (Taichung, Taiwan).

2.3. Study Design. We conducted a single-blind randomized, parallel, placebo-controlled study. The sampling and trial schemes are summarized in Figure 1. With a sample size calculation, we expected that the change in the levels of antioxidant enzymes activities would be $5.0 \pm 7.0$ units $/ \mathrm{mg}$ of protein after the WGP intervention; therefore, the desired power was set at 0.8 to detect a true effect and at an $\alpha$ value equal to 0.05 with a minimal sample size of 18 subjects in each intervention group. We enrolled 54 subjects with MS in this study that were randomly assigned to the placebo $(n=28)$ or WGP $(n=26)$ group. The female subjects in this study were postmenopausal women who were not receiving hormone therapy. The intervention was administered for 12 weeks, and the subjects were instructed to take eight capsules daily (WGP $4 \mathrm{~g} / \mathrm{d}$ and $0.5 \mathrm{~g} /$ capsule). To monitor compliance, the researchers reminded subjects to check the capsule bag every 4 weeks to confirm that the bag was empty. The age; blood pressure; and smoking, drinking, and exercise habits of all subjects were recorded. Body weight and height were measured; the body mass index $\left(\mathrm{kg} / \mathrm{m}^{2}\right)$ was then calculated.

2.4. Biochemical Measurement. Fasting venous blood samples $(15 \mathrm{~mL})$ were collected to estimate the hematological parameters. Blood specimens were collected in vacutainer tubes without EDTA as an anticoagulant. Serum was prepared and then frozen $\left(-80^{\circ} \mathrm{C}\right)$ for storage until analysis. The quantitative measurements of FG and blood lipid profiles [i.e., total cholesterol (TC), triglyceride (TG), LDL-C, and HDL$\mathrm{C}$ ] were measured using an automated biochemical analyzer (Hitachi-7180E, Tokyo, Japan). Red blood cells (RBCs) were diluted with $25 \mathrm{x}$ sodium phosphate buffer for superoxide dismutase (SOD) and glutathione peroxidase (GPx) measurements and with 250x sodium phosphate buffer for catalase (CAT) measurement. The methods for measuring CAT, SOD, and GPx were previously described [26-28]. The protein content of plasma and RBCs was determined based on the biuret reaction using a BCA kit (Thermo, Rockford, IL, USA). The antioxidant enzymes activities were expressed as units/mg of protein. All analyses were performed in duplicate.

2.5. Statistical Analyses. The data were analyzed using SigmaPlot software (version 12.0, Systat, San Jose, CA, USA). The normality of the distribution of the variables was evaluated using the Shapiro-Wilk test. Differences in subject demographics and hematological measurements between the placebo and WGP groups were analyzed by Student's $t$-test or the Mann-Whitney rank sum test. The paired $t$-test or Wilcoxon signed rank test was used to analyze the data within each group before (baseline) and after intervention (week 12). For categorical response variables, differences between the two groups were assessed by the Chi-square test or Fisher's exact test. To examine the correlations of blood pressure, FG, lipid profiles, and antioxidant enzymes activities after supplementation, the Pearson product moment correlation or Spearman rank order correlation was used. The data are 


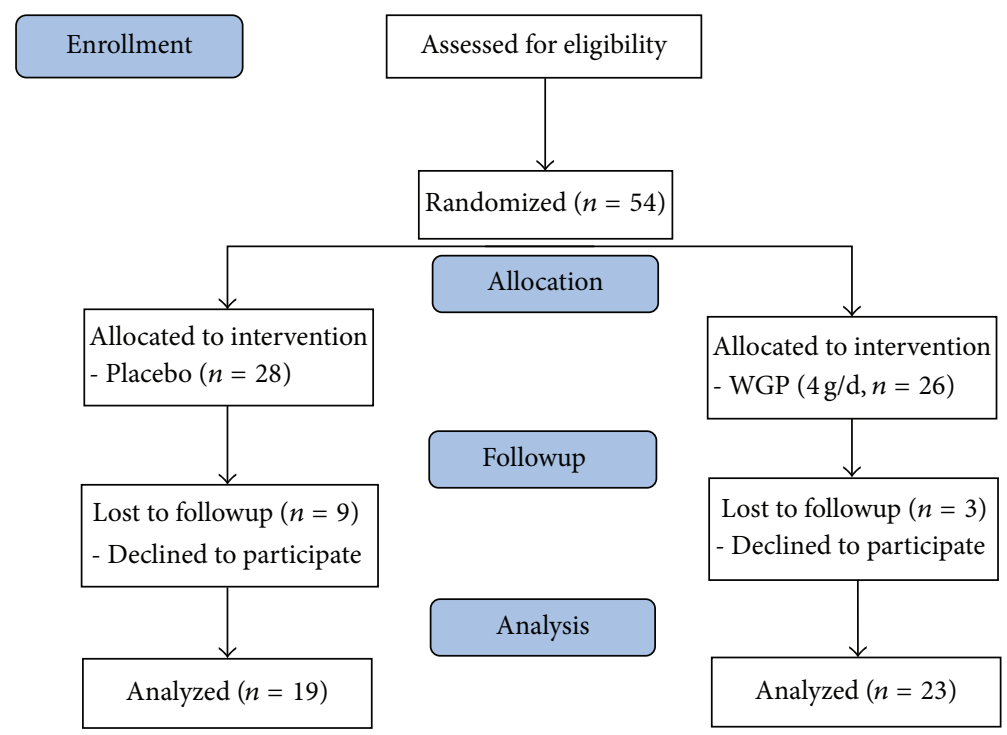

FIGURE 1: CONSORT scheme. WGP: water extracts of Graptopetalum paraguayense.

TABLE 1: Subjects characteristics.

\begin{tabular}{lccc}
\hline & Placebo $(n=19)$ & WGP $(n=23)$ & $P$ values \\
\hline Male/female $(n)$ & $12 / 7$ & $14 / 9$ & 0.88 \\
Age $(\mathrm{y})$ & $56.2 \pm 9.7(57.0)$ & $50.8 \pm 11.8(52.0)$ & 0.12 \\
Waist circumference $(\mathrm{cm})$ & $100.2 \pm 10.4(100.0)$ & $98.5 \pm 12.3(96.0)$ & 0.62 \\
Waist hip ratio & $0.9 \pm 0.1(0.9)$ & $29.3 \pm 0.1(0.9)$ & 0.87 \\
Body mass index $\left(\mathrm{kg} / \mathrm{m}^{2}\right)$ & $29.4 \pm 4.1(29.1)$ & $2(8.7 \%)$ & 0.80 \\
Current smoker $^{1}, n(\%)$ & $3(15.8 \%)$ & $2(8.7 \%)$ & 0.64 \\
Consuming alcohol $^{2}, n(\%)$ & $2(10.5 \%)$ & $14(61.9 \%)$ & 1.00 \\
Exercise $^{3}, n(\%)$ & $11(57.9 \%)$ & 0.90 \\
\hline
\end{tabular}

Data are given as mean \pm SD (median).

${ }^{1}$ Current smoker: individuals currently smoking one or more cigarettes per day.

${ }^{2}$ Consuming alcohol: individuals regularly drinking one or more alcoholic beverages per day.

${ }^{3}$ Exercise: individuals exercising regularly at least three times every week.

WGP: water extracts of Graptopetalum paraguayense.

expressed as the means \pm standard deviations (SD). The results were considered statistically significant at $P<0.05$.

\section{Results}

3.1. Characteristics of the Participants. Forty-two subjects completed the study (placebo, $n=19$; WGP, $n=23$ ). Table 1 presents the demographic data and health characteristics of the subjects. There were no significant differences between the two groups with respect to age; anthropometric measurements; and the frequency of smoking, drinking, or exercise at baseline.

3.2. Blood Pressure, FG, and Lipid Profiles after WGP Supplementation. Blood pressure, FG, and lipid profiles measurements are presented in Figures 2 and 3. The levels of SBP $(P<$ $0.01)$ and LDL-C $(P=0.06)$ were significantly decreased, while HDL-C was significantly increased $(P=0.01)$ after WGP supplementation. The subjects in the WGP group had significantly lower SBP $(P=0.01)$, FG $(P=0.04)$, TC $(P=0.08)$, TG $(P=0.07)$, and LDL-C $(P=0.04)$ levels and higher HDL-C $(P=0.01)$ level than those in the placebo group at week 12 .

3.3. Antioxidant Enzymes Activities after WGP Supplementation. The antioxidant enzymes activities are presented in Figure 4. The activities of CAT $(P<0.01)$ and SOD $(P=$ $0.02)$ were significantly increased after WGP supplementation. The subjects in the WGP group had significantly higher CAT and SOD activities than those in the placebo group at week $12(P<0.01)$.

3.4. Correlations between Blood Pressure, FG, Lipid Profiles, and Antioxidant Enzymes Activities after WGP Supplementation. The correlations between blood pressure, FG, lipid profiles, and antioxidant enzymes activities after 12 weeks of supplementation are presented in Table 2 . After 12 weeks of supplementation, the antioxidant enzymes activities were 
TABLE 2: Correlations $\left(r^{1}\right)$ between blood pressure, fasting glucose, lipid profiles, and antioxidant enzymes activities after 12 weeks of supplementation.

\begin{tabular}{|c|c|c|c|c|c|c|}
\hline & \multicolumn{2}{|c|}{ CAT (U/mg protein) } & \multicolumn{2}{|c|}{ SOD (U/mg protein) } & \multicolumn{2}{|c|}{ GPx (U/mg protein) } \\
\hline & Week 12 & $\Delta 12-0^{2}$ & Week 12 & $\Delta 12-0$ & Week 12 & $\Delta 12-0$ \\
\hline \multicolumn{7}{|c|}{$\mathrm{SBP}(\mathrm{mmHg})$} \\
\hline Week 12 & $-0.41^{*}$ & & -0.10 & & $-0.26^{*}$ & \\
\hline$\Delta 12-0$ & & -0.07 & & 0.09 & & -0.19 \\
\hline \multicolumn{7}{|c|}{$\mathrm{DBP}(\mathrm{mmHg})$} \\
\hline Week 12 & $-0.25^{*}$ & & $-0.17^{*}$ & & $-0.28^{*}$ & \\
\hline$\Delta 12-0$ & & -0.04 & & -0.11 & & $-0.25^{*}$ \\
\hline \multicolumn{7}{|c|}{$\mathrm{FG}(\mathrm{mmol} / \mathrm{L})$} \\
\hline Week 12 & $-0.01^{*}$ & & $-0.01^{*}$ & & $-0.01^{*}$ & \\
\hline$\Delta 12-0$ & & $9.44 \times 10^{-3}$ & & $1.11 \times 10^{-3}$ & & $3.89 \times 10^{-3}$ \\
\hline \multicolumn{7}{|c|}{$\mathrm{TC}(\mathrm{mmol} / \mathrm{L})$} \\
\hline Week 12 & $-4.14 \times 10^{-3}$ & & 0.00 & & $-2.59 \times 10^{-4}$ & \\
\hline$\Delta 12-0$ & & $-7.25 \times 10^{-3 *}$ & & $-1.55 \times 10^{-3}$ & & $-6.99 \times 10^{-3 *}$ \\
\hline \multicolumn{7}{|c|}{$\mathrm{TG}(\mathrm{mmol} / \mathrm{L})$} \\
\hline Week 12 & $-1.70 \times 10^{-3}$ & & $-2.26 \times 10^{-3}$ & & $-1.36 \times 10^{-3}$ & \\
\hline$\Delta 12-0$ & & $-3.16 \times 10^{-3 *}$ & & $-4.41 \times 10^{-3 *}$ & & $-4.52 \times 10^{-3 *}$ \\
\hline \multicolumn{7}{|c|}{ LDL-C (mmol/L) } \\
\hline Week 12 & $-3.77 \times 10^{-3}$ & & $-4.14 \times 10^{-3}$ & & $-3.63 \times 10^{-3}$ & \\
\hline$\Delta 12-0$ & & $1.04 \times 10^{-3}$ & & $3.89 \times 10^{-3}$ & & $-7.77 \times 10^{-4}$ \\
\hline \multicolumn{7}{|c|}{$\mathrm{HDL}-\mathrm{C}(\mathrm{mmol} / \mathrm{L})$} \\
\hline Week 12 & $0.01^{*}$ & & $7.25 \times 10^{-3 *}$ & & $1.55 \times 10^{-3}$ & \\
\hline$\Delta 12-0$ & & 0.00 & & $0.01^{*}$ & & $3.89 \times 10^{-3}$ \\
\hline
\end{tabular}

${ }^{1}$ Correlation coefficient.

${ }^{2} \Delta 12-0$, changes from week 0 to week 12 .

${ }^{*} P<0.05$.

CAT: catalase; FG: fasting glucose; GPx: glutathionine peroxidase; HDL-C: high density lipoprotein-cholesterol; LDL-C: low density lipoprotein-cholesterol; SOD: superoxide dismutase; TC: total cholesterol; TG: triglyceride; WGP: water extracts of Graptopetalum paraguayense.
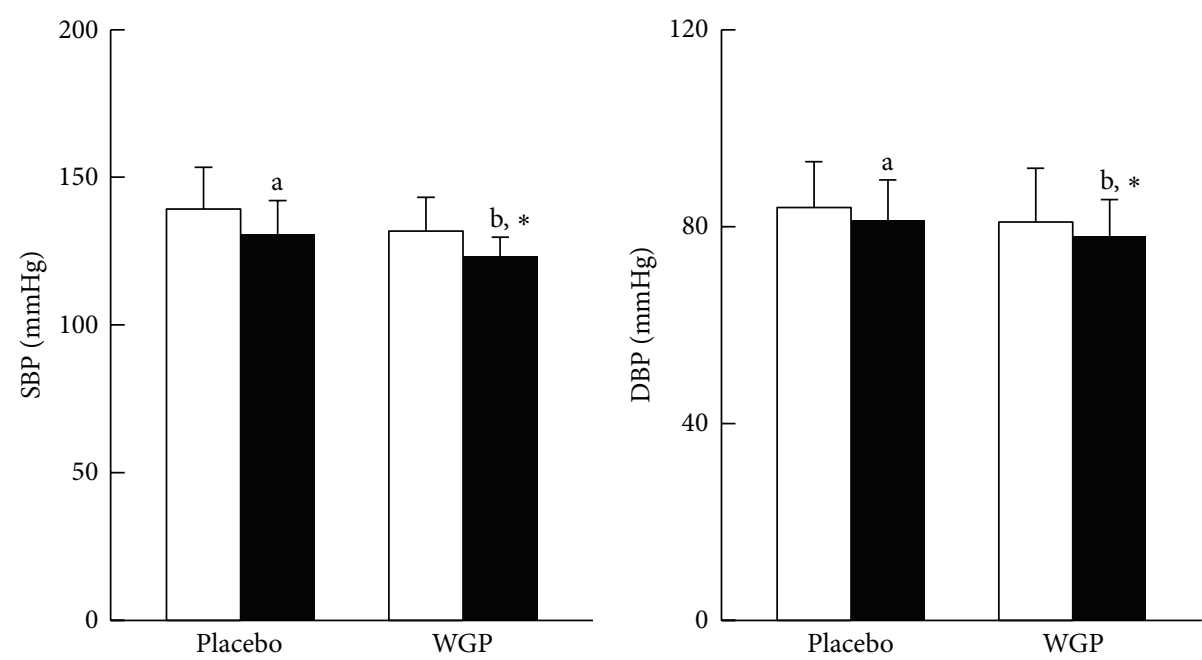

FIGURE 2: Blood pressure measurements. Data are means \pm SD. $\square$ Week 0 , $\square$ Week $12 .{ }^{*}$ Values were significantly different after intervention within the group, $P<0.05$. ${ }^{\mathrm{a}, \mathrm{b}}$ Values with different superscripts were significantly different between two groups, $P<0.05$. DBP: diastolic blood pressure; SBP: systolic blood pressure; WGP: water extracts of Graptopetalum paraguayense. 

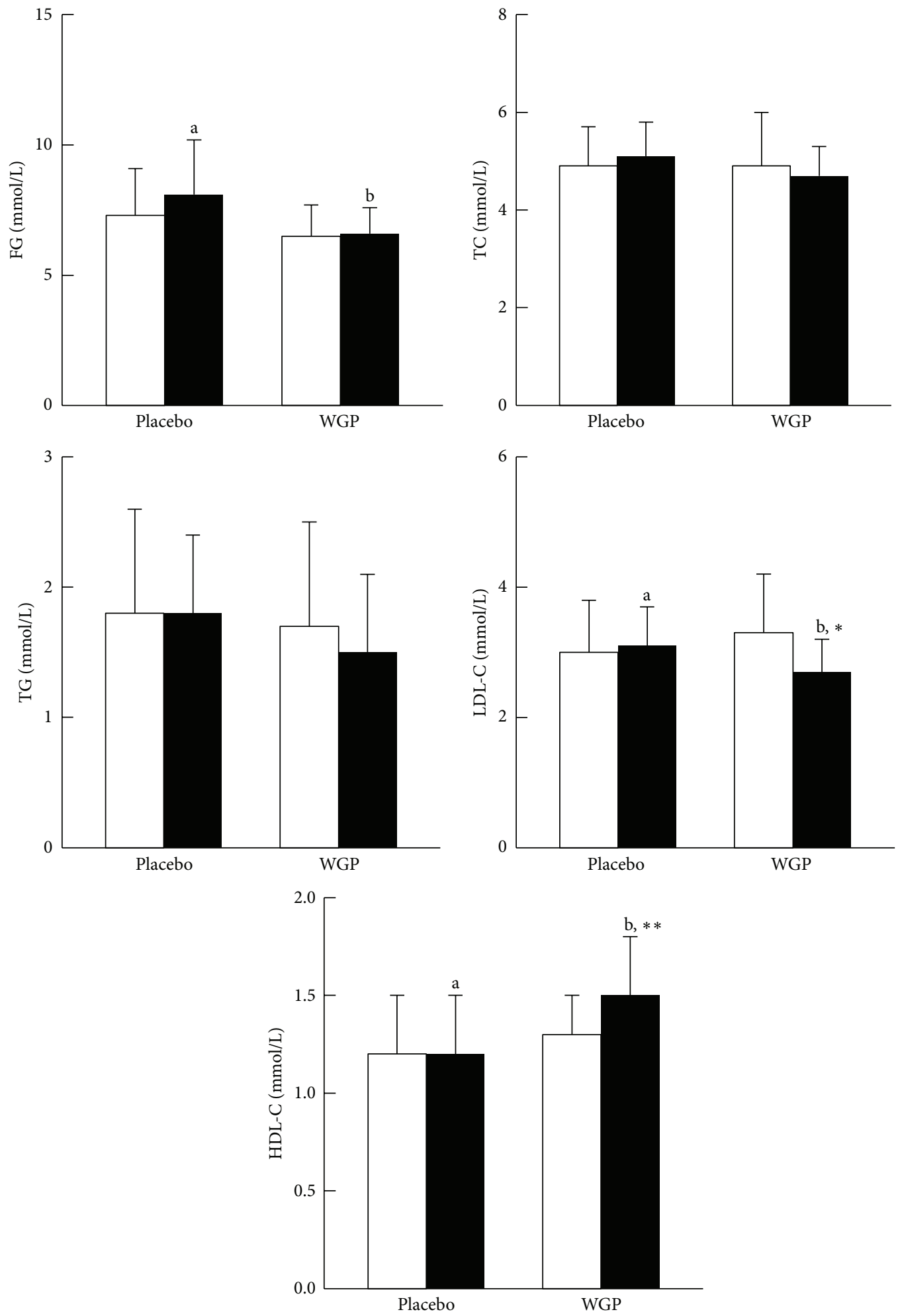

FIGURE 3: Fasting glucose level and blood lipid profiles. Data are means \pm SD. $\square$ Week 0 , $\square$ Week $12 .{ }^{*}$ Values were significantly different after intervention within the group; ${ }^{*} P=0.06 ;{ }^{* *} P=0.01$. ${ }^{\mathrm{a}, \mathrm{b}}$ Values with different superscripts were significantly different between two groups, $P<0.05$. FG: fasting glucose; HDL-C: high density lipoprotein cholesterol; LDL-C: low density lipoprotein cholesterol; TC: total cholesterol; TG: triglyceride; WGP: water extracts of Graptopetalum paraguayense. 

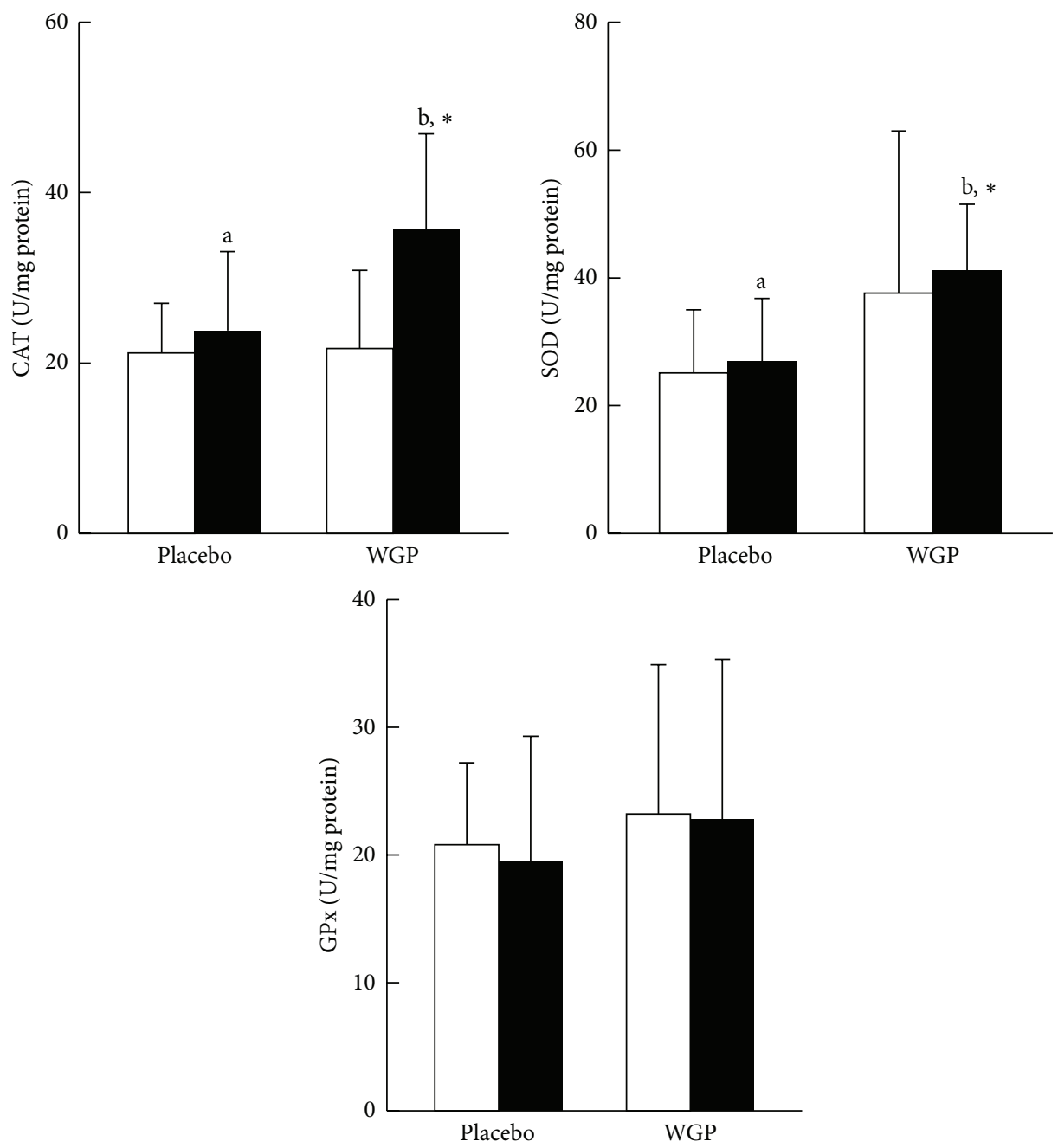

Figure 4: Antioxidant enzymes activities. Data are means \pm SD. $\square$ Week 0 , $\square$ Week $12 .{ }^{*}$ Values were significantly different after intervention within the group, $P<0.05$. ${ }^{\mathrm{a}, \mathrm{b}}$ Values with different superscripts were significantly different between two groups, $P<0.05$. CAT: catalase; GPx: glutathione peroxidase; SOD: superoxide dismutase; WGP: water extracts of Graptopetalum paraguayense.

significantly negatively correlated with the SBP, DBP, and FG level and positively correlated with HDL-C level $(P<0.05)$. The change in antioxidant enzymes activities was significantly correlated with the change in DBP and lipid profiles levels (TC, TG, and HDL-C, $P<0.05$ ).

3.5. Adverse Events. There were no clinically significant changes in the subjects' vital signs, serum chemical values, or hematological values, and there were no serious adverse events in either group.

\section{Discussion}

This is the first clinical study to report a statistically significant link between the components of MS and antioxidant status in MS subjects treated with WGP. In the present study, we observed that the characteristics of MS (blood pressure, FG, and lipid profiles) were significantly correlated with the antioxidant status (Table 2) after 12 weeks of WGP supplementation. The antioxidant capacity of Graptopetalum paraguayense has been demonstrated in both in vitro and in vivo studies [14-22]. Oxidative stress is thought to play an important role in the development of MS [9]. In general, individuals with MS are typically abdominally obese. Obesity is an oxidative burden that may lead to the reduction of antioxidant enzymes activities [29]. Antioxidant enzymes are the first line of defense against reactive oxygen species (ROS) and lead to a decrease in their activities [12, 30]. In the present study, the activities of CAT and SOD were significantly increased by $51 \%$ and $28 \%$, respectively, after 12 weeks of WGP supplementation. Plant phenol and polyphenolic compounds, such as flavonoids and anthocyanins, are widely distributed in natural herb and spice extracts, and they possess significant antioxidant activities [30,31]. Chung et al. reported that the total phenolic compounds and anthocyanins in Graptopetalum paraguayense extracts ranged from 11.0 to $34.0 \mathrm{mg} / \mathrm{g}$ and from 0.03 to $1.29 \mu \mathrm{mol} / \mathrm{g}$, respectively; these compounds may increase the radical-scavenging ability 
[15]. As a result, WGP may contribute to the first line of antioxidation, leading to increases in antioxidant status.

Hypertension and hyperglycemia induce the activation of oxidative stress pathways and mediate the development of cardiovascular disease and diabetes $[2,32]$. In this study, we treated subjects with MS using WGP at dose of $4 \mathrm{~g}$ daily for 12 weeks; SBP and FG levels were significantly decreased by $8 \%$ and $3 \%$, respectively, after treatment. Similar results were also found in animals models [17, 33]. For hypertension treatment, the inhibition of angiotensin converting enzyme (ACE) is a major modern therapeutic approach. The extracts of Graptopetalum paraguayense have demonstrated an antihypertension effect because of their inhibitory effects on ACE activity [17]. With respect to glucose regulation, pancreatic $\beta$-cells are sensitive to ROS and the peroxidation of $\beta$-cells may amplify the glucotoxicity and lipotoxicity effects of hyperglycemia [34]. Therefore, antioxidants, such as WGP, may exhibit a protective effect against oxidative stress by neutralizing ROS and improving insulin sensitivity in subjects with MS.

Previous studies on lipid metabolism have demonstrated that human serum paraoxonase (PON 1) may inhibit the oxidation of LDL-C and exclusively bind to HDL-C [3537]. After 12 weeks of WGP supplementation, LDL-C level was significantly decreased by $16 \%$ and HDL-C level was significantly increased by $10 \%$. Graptopetalum paraguayense could modulate the lipid metabolism by increasing PON 1 gene expression [38]. PON 1 can hydrolyze lipid peroxides in oxidized lipoproteins and atherosclerotic lesions [39]. In addition, Graptopetalum paraguayense might contain flavonoids and dietary fiber, which are important mediators in the regulation of lipid metabolism in MS and obesity $[40,41]$.

Lin et al. [22] reported that Graptopetalum paraguayense was related to side effects including diarrhea and oral ulcers. However, no subjects reported these side effects in the present study, possibly because the Graptopetalum paraguayense was administered as an oral supplement in capsule form. There were no clinically significant changes in the subjects' vital signs, serum chemical values, or hematological values, and no subject withdrew because of adverse events. Thus, WGP at a dose of $4 \mathrm{~g} / \mathrm{d}$ is safe for individuals with MS.

The population size is a limitation of the present study. The number of participants was small, although we recruited more subjects than expected. Large studies are needed to establish the beneficial effects of WGP supplementation.

In conclusion, subjects suffering from MS may exhibit a higher level of oxidative stress. The present study demonstrated a significant reduction in blood pressure, blood glucose, and lipid profiles and an increase in the antioxidant enzymes activities in subjects with MS after WGP supplementation. WGP might exert a beneficial effect on MS related to its antioxidative capacity.

\section{Conflict of Interests}

The authors have no conflict of interests.

\section{Acknowledgments}

The authors would like to express their sincere appreciation to the subjects for their participation. The authors also thank the nurses at Chung Shan Medical University Hospital for providing expert assistance with blood sample collection and data analysis.

\section{References}

[1] J. I. Cleeman, "Executive summary of the third report of the National Cholesterol Education Program (NCEP) expert panel on detection, evaluation, and treatment of high blood cholesterol in adults (adult treatment panel III)," The Journal of the American Medical Association, vol. 285, no. 19, pp. 24862497, 2001.

[2] E. Ferrannini, S. M. Haffner, B. D. Mitchell, and M. P. Stern, "Hyperinsulinaemia: the key feature of cardiovascular and metabolic syndrome," Diabetologia, vol. 34, no. 6, pp. 416-422, 1991.

[3] E. S. Ford and W. H. Giles, "A comparison of the prevalence of the metabolic syndrome using two proposed definitions," Diabetes Care, vol. 26, no. 3, pp. 575-581, 2003.

[4] Y.-W. Park, S. Zhu, L. Palaniappan, S. Heshka, M. R. Carnethon, and S. B. Heymsfield, "The metabolic syndrome: prevalence and associated risk factor findings in the US population from the Third National Health and Nutrition Examination Survey, 1988-1994," Archives of Internal Medicine, vol. 163, no. 4, pp. 427-436, 2003.

[5] G. D. Kolovou, K. K. Anagnostopoulou, K. D. Salpea, and D. P. Mikhailidis, "The prevalence of metabolic syndrome in various populations," The American Journal of the Medical Sciences, vol. 333, no. 6, pp. 362-371, 2007.

[6] C.-J. Yeh, H.-Y. Chang, and W.-H. Pan, "Time trend of obesity, the metabolic syndrome and related dietary pattern in Taiwan: from NAHSIT 1993-1996 to NAHSIT 2005-2008," Asia Pacific Journal of Clinical Nutrition, vol. 20, no. 2, pp. 292-300, 2011.

[7] A. Ceriello, A. Quatraro, and D. Giugliano, "Diabetes mellitus and hypertension: the possible role of hyperglycaemia through oxidative stress," Diabetologia, vol. 36, no. 3, pp. 265-266, 1993.

[8] D. Giugliano, A. Ceriello, and G. Paolisso, "Diabetes mellitus, hypertension, and cardiovascular disease: which role for oxidative stress?" Metabolism, vol. 44, no. 3, pp. 363-368, 1995.

[9] I. C. West, "Radicals and oxidative stress in diabetes," Diabetic Medicine, vol. 17, no. 3, pp. 171-180, 2000.

[10] C. Antoniades, D. Tousoulis, C. Tentolouris, P. Toutouzas, and C. Stefanadis, "Oxidative stress, antioxidant vitamins, and atherosclerosis: from basic research to clinical practice," Herz, vol. 28, no. 7, pp. 628-638, 2003.

[11] R. Stocker and J. F. Keaney Jr., "Role of oxidative modifications in atherosclerosis," Physiological Reviews, vol. 84, no. 4, pp. 13811478, 2004.

[12] S. Penckofer, D. Schwertz, and K. Florczak, "Oxidative stress and cardiovascular disease in type 2 diabetes: the role of antioxidants and pro-oxidants," The Journal of Cardiovascular Nursing, vol. 16, no. 2, pp. 68-85, 2002.

[13] D. Giugliano, "Dietary antioxidants for cardiovascular prevention," Nutrition, Metabolism and Cardiovascular Diseases, vol. 10, no. 1, pp. 38-44, 2000.

[14] K.-F. Huang, Y.-W. Chen, C.-T. Chang, and S.-T. Chou, "Studies on the inhibitory effect of Graptopetalum paraguayense E. 
Walther extracts on mushroom tyrosinase," Food Chemistry, vol. 89, no. 4, pp. 583-587, 2005.

[15] Y.-C. Chung, S.-J. Chen, C.-K. Hsu, C.-T. Chang, and S.-T. Chou, "Studies on the antioxidative activity of Graptopetalum paraguayense E. Walther," Food Chemistry, vol. 91, no. 3, pp. 419424, 2005.

[16] S.-J. Chen, C.-T. Chang, Y.-C. Chung, and S.-T. Chou, "Studies on the inhibitory effect of Graptopetalum paraguayense E. Walther extracts on the angiotensin converting enzyme," Food Chemistry, vol. 100, no. 3, pp. 1032-1036, 2007.

[17] Y.-C. Chung, S.-J. Chen, H.-Y. Pengspi-Sup, and S.-T. Chou, "Anti hypertensive and antioxidant effects of the Graptopetalum paraguayense E. Walther extract in spontaneously hypertensive rats," Journal of the Science of Food and Agriculture, vol. 89, no. 15, pp. 2678-2686, 2009.

[18] S.-J. Chen, J.-G. Chung, Y.-C. Chung, and S.-T. Chou, "In vitro antioxidant and antiproliferative activity of the stem extracts from Graptopetalum paraguayense," American Journal of Chinese Medicine, vol. 36, no. 2, pp. 369-383, 2008.

[19] P.-D. Duh, S.-L. Lin, and S.-C. Wu, "Hepatoprotection of Graptopetalum paraguayense E. Walther on $\mathrm{CCl} 4$-induced liver damage and inflammation," Journal of Ethnopharmacology, vol. 134, no. 2, pp. 379-385, 2011.

[20] L. J. Su, C. C. Chang, C. H. Yang et al., "Graptopetalum paraguayense ameliorates chemical-induces rat hepatic fibrosis in vivo and inactivates stellate cells and kupffer cells in vitro," PLoS ONE, no. 8, Article ID e53988, 2013.

[21] T.-K. Kao, Y.-C. Ou, S.-L. Raung et al., "Graptopetalum paraguayense E. Walther leaf extracts protect against brain injury in ischemic rats," American Journal of Chinese Medicine, vol. 38, no. 3, pp. 495-516, 2010.

[22] Y. L. Lin, H. Y. Peng, H. M. Hsieh, C. H. Lin, and S. T. Chou, "Effects of Graptopetalum paraguayense consumption on serum lipid profiles and antioxidative status in hypercholesteremic subjects," Journal of the Science of Food and Agriculture, vol. 91, no. 7, pp. 1230-1235, 2011.

[23] K. G. M. M. Alberti and P. Zimmet, "The metabolic syndromea new worldwide definition," The Lancet, vol. 366, no. 9491, pp. 1059-1062, 2005.

[24] S. M. Grundy, J. I. Cleeman, S. R. Daniels et al., "Diagnosis and management of the metabolic syndrome: an American Heart Association/National Heart, Lung, and Blood Institute scientific statement," Circulation, vol. 112, no. 17, pp. 2735-2752, 2005.

[25] Y.-C. Chung, S.-T. Chou, J.-K. Jhan, J.-W. Liao, and S.-J. Chen, "In vitro and in vivo safety of aqueous extracts of Graptopetalum paraguayense E. Walther," Journal of Ethnopharmacology, vol. 140, no. 1, pp. 91-97, 2012.

[26] D. E. Paglia and W. N. Valentine, "Studies on the quantitative and qualitative characterization of erythrocyte glutathione peroxidase," The Journal of Laboratory and Clinical Medicine, vol. 70, no. 1, pp. 158-169, 1967.

[27] S. Marklund and G. Marklund, "Involvement of the superoxide anion radical in the autoxidation of pyrogallol and a convenient assay for superoxide dismutase," European Journal of Biochemistry, vol. 47, no. 3, pp. 469-474, 1974.

[28] H. Aebi, "[13] Catalase in vitro," Methods in Enzymology, vol. 105, no. C, pp. 121-126, 1984.

[29] N. Karaouzene, H. Merzouk, M. Aribi et al., "Effects of the association of aging and obesity on lipids, lipoproteins and oxidative stress biomarkers: a comparison of older with young men," Nutrition, Metabolism and Cardiovascular Diseases, vol. 21, no. 10, pp. 792-799, 2011.
[30] S. A. B. E. Van Acker, D.-J. Van Den Berg, M. N. J. L. Tromp et al., "Structural aspects of antioxidant activity of flavonoids," Free Radical Biology \& Medicine, vol. 20, no. 3, pp. 331-342, 1996.

[31] W. Zheng and S. Y. Wang, "Antioxidant activity and phenolic compounds in selected herbs," Journal of Agricultural and Food Chemistry, vol. 49, no. 11, pp. 5165-5170, 2001.

[32] J. L. Evans, I. D. Goldfine, B. A. Maddux, and G. M. Grodsky, "Are oxidative stress-activated signaling pathways mediators of insulin resistance and $\beta$-cell dysfunction?" Diabetes, vol. 52, no. 1, pp. 1-8, 2003.

[33] J. I. Yang, M. H. Pan, M. L. Tsai, and W. C. Chen, "Effects of Shilan (Graptopetalum paraguayens) extracts on antioxidative performance and serum glucose level in Streptozotocin-induced diabetic rats," Journal of National Kaohsiung Marine University, vol. 18, pp. 119-132, 2003 (Chinese).

[34] V. Poitout and R. P. Robertson, "Minireview: secondary $\beta$-cell failure in type 2 diabetes-a convergence of glucotoxicity and lipotoxicity," Endocrinology, vol. 143, no. 2, pp. 339-342, 2002.

[35] M.-C. Blatter, R. W. James, S. Messner, F. Barja, and D. Pometta, "Identification of a distinct human high-density lipoprotein subspecies defined by a lipoprotein-associated protein, K45. Identity of K-45 with paraoxonase," European Journal of Biochemistry, vol. 211, no. 3, pp. 871-879, 1993.

[36] M. N. Rao, Q.-H. Liu, P. Marmillot, L. B. Seeff, D. B. Strader, and M. R. Lakshman, "High-density lipoproteins from human alcoholics exhibit impaired reverse cholesterol transport function," Metabolism, vol. 49, no. 11, pp. 1406-1410, 2000.

[37] F. Osaki, Y. Ikeda, T. Suehiro et al., "Roles of Sp1 and protein kinase $\mathrm{C}$ in regulation of human serum paraoxonase 1 (PON1) gene transcription in HepG2 cells," Atherosclerosis, vol. 176, no. 2, pp. 279-287, 2004.

[38] C.-C. Cheng, L.-C. Wu, J.-M. Lai, C.-T. Chen, C.-M. Hsueh, and S.-L. Hsu, "Ethanol extract of Graptopetalum paraguayense upregulates paraoxonase 1 gene expression via an AKT/NF- $\kappa \mathrm{B}-$ dependent pathway," American Journal of Chinese Medicine, vol. 40, no. 2, pp. 357-372, 2012.

[39] B. Mackness, P. Durrington, A. Povey et al., "Paraoxonase and susceptibility to organophosphorus poisoning in farmers dipping sheep," Pharmacogenetics, vol. 13, no. 2, pp. 81-88, 2003.

[40] A. Papathanasopoulos and M. Camilleri, "Dietary fiber supplements: effects in obesity and metabolic syndrome and relationship to gastrointestinal functions," Gastroenterology, vol. 138, no. 1, pp. 65-e2, 2010.

[41] J. M. Assini, E. E. Mulvihill, and M. W. Huff, "Citrus flavonoids and lipid metabolism," Current Opinion in Lipidology, vol. 24, no. 1, pp. 34-40, 2013. 


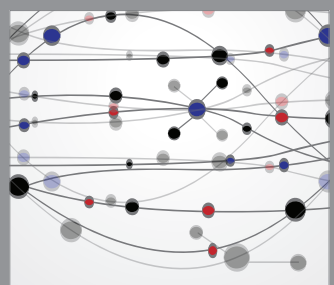

The Scientific World Journal
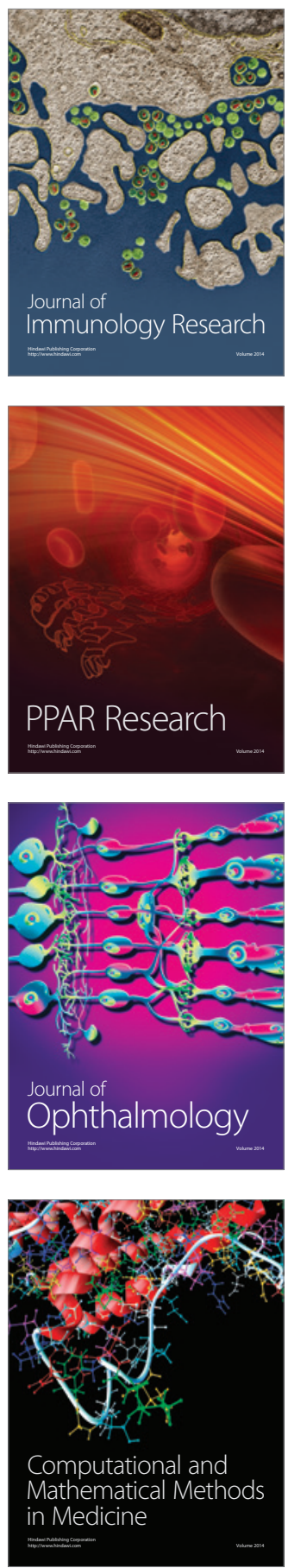

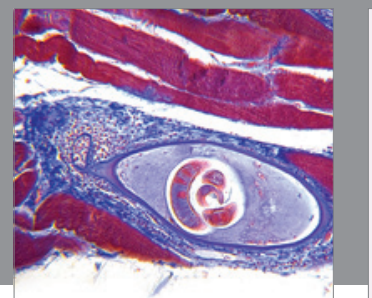

Gastroenterology

Research and Practice
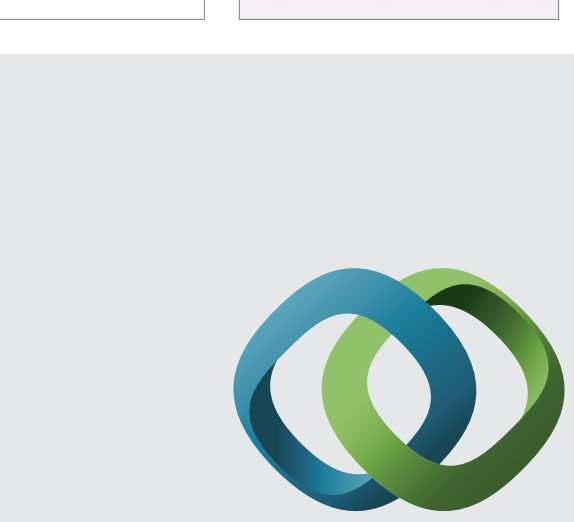

\section{Hindawi}

Submit your manuscripts at

http://www.hindawi.com
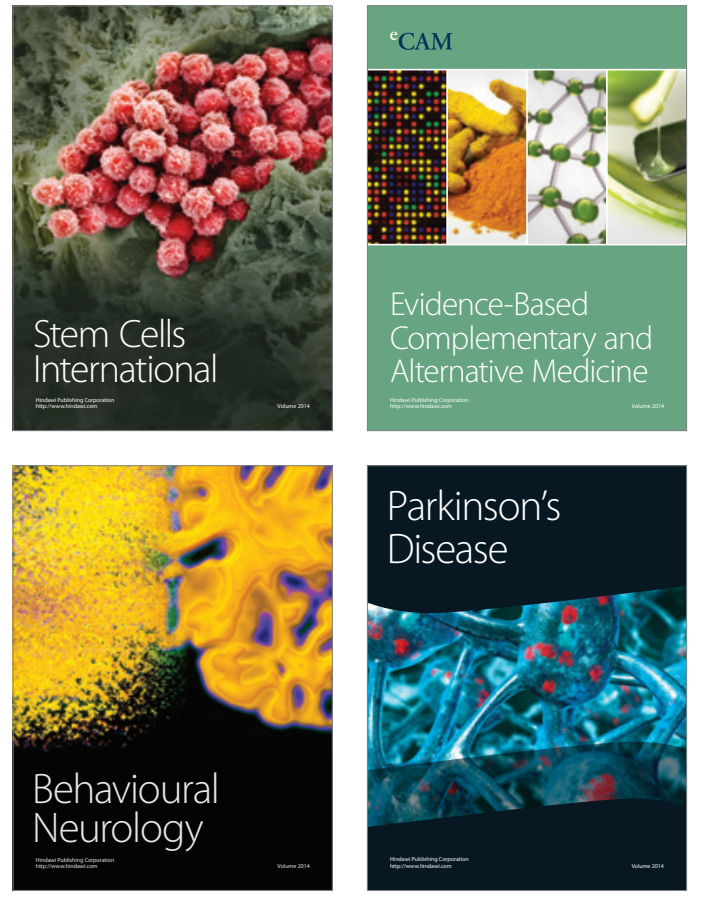
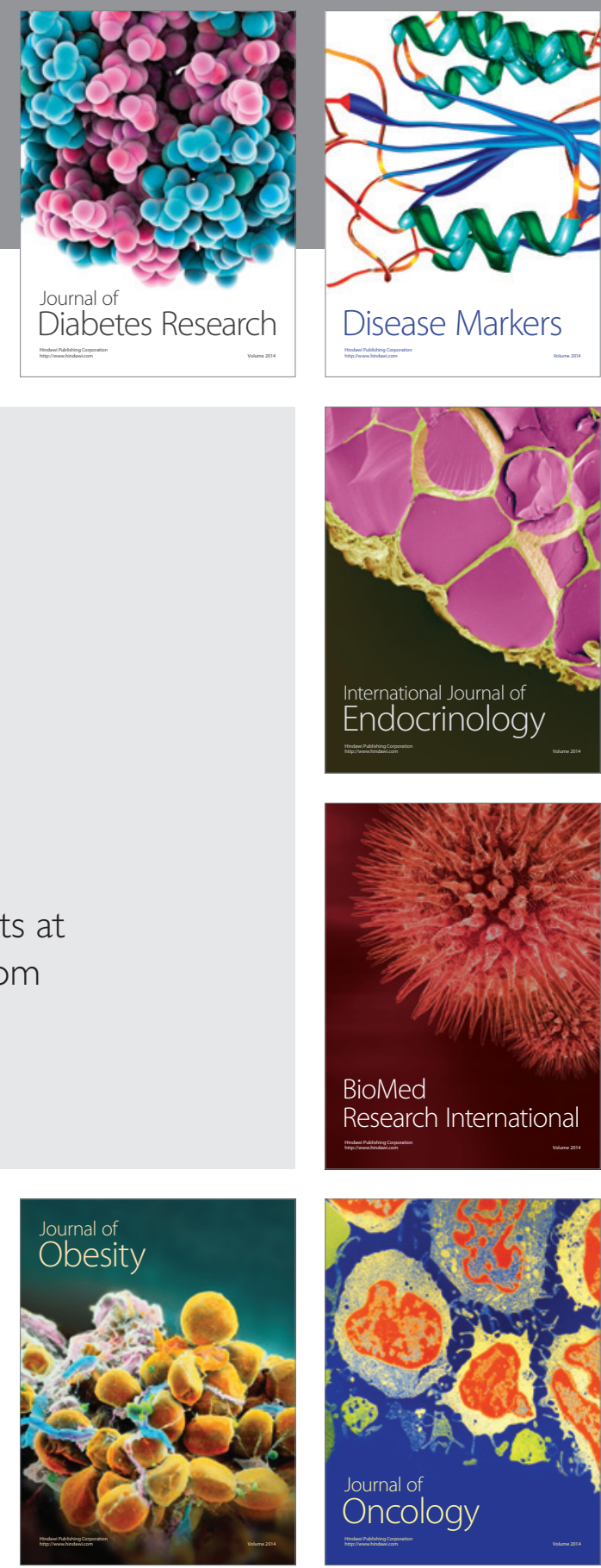

Disease Markers
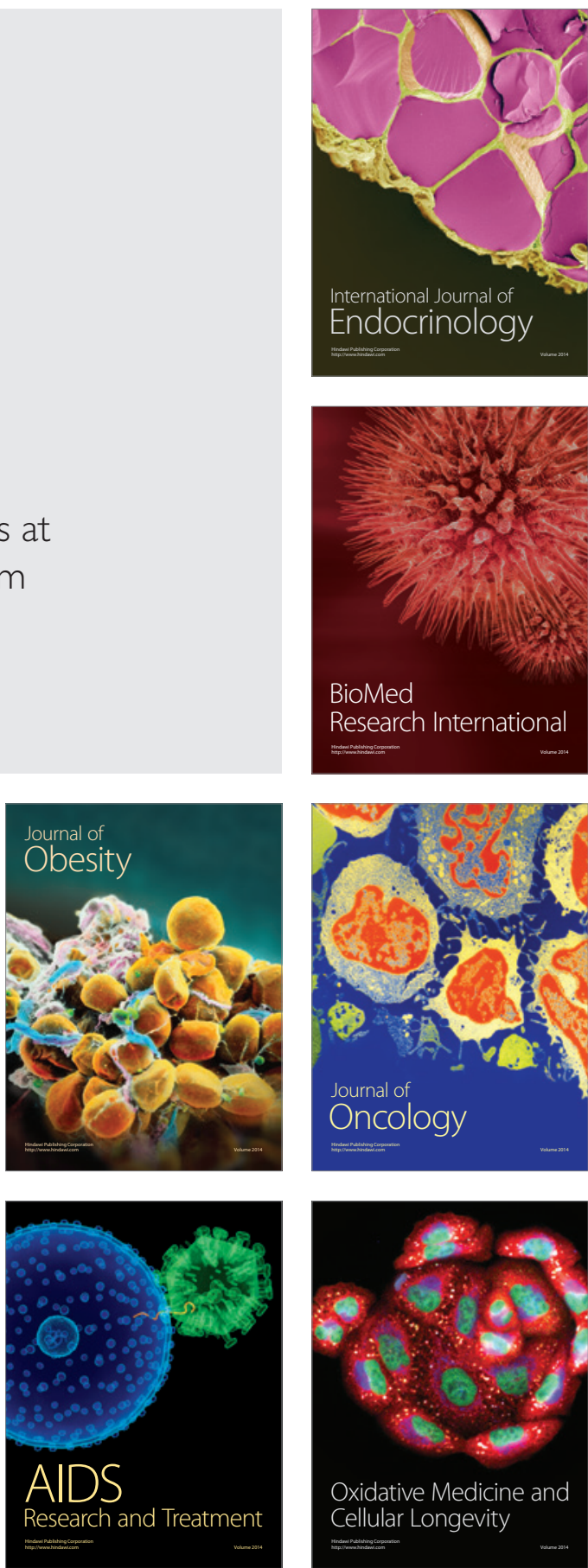